\title{
Relationships of youth risk behaviors with norm-consciousness and resilience among Japanese high school students
}

\author{
Chie Kataoka $^{1^{*}}$, Yuji Nozu ${ }^{1}$, Shiori Kawahara ${ }^{2}$, Hiroko Kuroiwa ${ }^{2}$, Masako Kudo ${ }^{2}$, Yuki Sato ${ }^{3}$, \\ Motoyoshi Kubo ${ }^{4}$, Motoi Watanabe ${ }^{5}$ \\ ${ }^{1}$ Faculty of Health and Sport Sciences, University of Tsukuba, Tsukuba, Japan; *Corresponding Author: kataoka@taiiku.tsukuba.ac.jp \\ ${ }^{2}$ Graduate School of Comprehensive Human Sciences, University of Tsukuba, Tsukuba, Japan \\ ${ }^{3}$ Tomiya Junior High School, Sendai, Japan \\ ${ }^{4}$ Faculty of Education, Utsunomiya University, Utsunomiya, Japan \\ ${ }^{5}$ Hokkaido University of Education, Sapporo, Japan
}

Received 21 March 2012; revised 26 April 2012; accepted 18 May 2012

\section{ABSTRACT}

The purpose of this study was to examine the influences of norm-consciousness and resilience on the prevention of youth risk behaviors among Japanese high school students. Data of a national survey, Japan Youth Risk Behavior Survey 2011, was used for this analysis. The subjects were 9778 students (5027 males, 4751 females) in grades 10 - 12 in 102 schools randomly selected from among high schools throughout Japan. When multiple regression analysis was carried out using four subdomains of norm-consciousness and three subdomains of resilience as the independent variables and nine risk behaviors as the dependent variables, the subdomains of norm-consciousness showed significant positive standardized partial regression coefficients for eight risk behaviors for both males and females. In particular, "current alcohol use" and "ever had sexual intercourse" showed the largest standardized partial regression coefficients for the norm-consciousness in school, and "current cigarette use" showed that for the norm-consciousness in community. The subdomains of resilience showed significant positive standardized partial regression coefficients for five risk behaviors for males and for six those for females. The results of this study suggest that the norm-consciousness is an important protective factor to comprehensively prevent risk behaviors among Japanese adolescents. It is expected to be effective to enhance the norm-consciousness particularly in school and community. In addition, resilience should also be emphasized as a factor to prevent specific risk behaviors.
Keywords: Youth Risk Behavior; Norm-Consciousness; Resilience; Japan

\section{INTRODUCTION}

Among adolescents in Japan and overseas, the problems of health-risk behaviors, which seriously affect their lives and health, are pointed out and the prevention is one of the important social issues. Recently, studies in which such risk behaviors are comprehensively taken into consideration have been reported. In the Centers for Disease Control and Prevention (CDC) [1] in the US, the Youth Risk Behavior Surveillance System (YRBSS) monitors the following six categories: 1) behaviors that contribute to unintentional injuries and violence; 2) tobacco use; 3) alcohol and other drug use; 4) sexual behaviors that contribute to unintended pregnancy and sexually transmitted diseases (STDs), including human immunodeficiency virus (HIV) infection; 5) unhealthy dietary behaviors; and 6) physical inactivity. The CDC conducted the surveys in 1990 and every two years since 1991, and reported the results. The prevalence of youth risk behaviors is investigated on a nationwide scale also in Romania [2], the Republic of South Africa [3], South Korea [4], and other countries. In Japan, Yuji Nozu [5] and colleagues conducted Japan Youth Risk Behavior Survey, a national survey in 2001, and they have reported the prevalence of risk behaviors and the relationships among risk behaviors.

To prevent youth risk behaviors, it is necessary to clarify the factors related to risk behaviors. In school health education, which plays an important role in the prevention of youth risk behaviors, the factors that are expected to be improved by educational intervention should be particularly emphasized. To comprehensively prevent risk behaviors, it is considered meaningful to 
emphasize psychosocial factors commonly related to risk behaviors.

Among Japanese adolescents, it is pointed out that norm-consciousness is one of the important psychosocial factors that are protective in relation to risk behaviors. It has been reported that norm-consciousness is particularly strongly related to risk behaviors that are illegal in Japan, such as, drug abuse and smoking and drinking among young people aged 20 years or younger [6,7]. In addition, norm-consciousness is considered as an individual value consciousness to respect and agree with norms [8].

On the other hand, resilience has attracted attention as a psychosocial factor related to youth risk behaviors. Resilience is a concept that has been noticed by people under markedly adverse circumstances, such as poverty and alcohol dependence. For example, Masten et al. [9] defined resilience as follows: "Resilience refers to the process of, capacity for, or outcome of successful adaptation despite challenging or threatening circumstances”. Regarding the resilience among adolescents, studies are progressed centered on Western countries and the US and the policy to enhance their resilience is considered [10]. Currently, Japanese adolescents face adverse circumstances; for instance, $14.2 \%$ of adolescents aged 17 years or younger are in relative poverty [11], the unemployment rate of those aged 15 - 19 years is 9.8\% [12], and many were affected by the Great East Japan Earthquake in 2011. It is significant to focus on resilience of today's adolescents in Japan as a factor related to the prevalence of their risk behaviors.

In this study, we aim to examine the influences of norm-consciousness and resilience on the prevention of risk behaviors among Japanese high school students by comprehensively focusing on youth risk behaviors.

\section{METHODS}

\subsection{Analysis Data}

The data of Japan Youth Risk Behavior Survey (JYRBS 2011), a national survey conducted using an anonymous self-administered questionnaire, was used for this analysis. Analysis subjects were 9778 students (5027 males and 4751 females) in grades 10 - 12 in 102 schools randomly selected from among high schools throughout Japan. In addition, this survey was reviewed and approved by the research ethics committee of Graduate School of Comprehensive Human Sciences, University of Tsukuba.

In this study, we used nine questions of risk behaviors and two measurement scales (norm-consciousness scale and resilience scale) from among the survey items of JYRBS 2011. The survey has a total of 41 questions on nine categories of risk behaviors: 1) physical activity; 2) dietary behaviors; 3) cigarette smoking; 4) alcohol drinking; 5) drug abuse; 6) sexual behaviors; 7) traffic-related behaviors; 8) violence and carrying a weapon; and 9) self-harm behaviors. Nine questions used in this study are as follows: "lack of vigorous physical activity" (prevalences in JYRBS 2011: male, 40.0\%; female, 63.5\%), "skipping breakfast" (male, 26.9\%; female, 26.0\%), "current cigarette use" (smoked cigarettes on at least 1 day during the 30 days before the survey). (male, 6.0\%; female, 3.1\%), "current alcohol use" (had at least one drink of alcohol on at least 1 day during the 30 days before the survey). (male, 16.6\%; female, 16.1\%), "lifetime thinner use" (male, 0.9\%; female, 0.5\%), "ever had sexual intercourse" (male, 15.9\%; female, 19.4\%), "rarely or never wore seatbelts” (male, 21.0\%; female, 20.9\%), “in a physical fight" (male, $12.0 \%$; female, 6.7\%), and "seriously considered attempting suicide” (male, 16.3\%; female, 27.8\%).

Norm-consciousness scale comprises a total of 12 items with the following four subdomains: norm-consciousness at home, norm-consciousness in school, normconsciousness in community, and norm-consciousness among peers. This scale was prepared considering that norms are different among places and situations that adolescents are closely related to in their daily lives, such as home, school, local community, and peers, and the reliability and validity of the scale were confirmed by Chie Uehara et al. [13].

Resilience scale comprises a total of 21 items with the following three subdomains: Novelty Seeking, Emotional Regulation, and Positive Future Orientation. This scale was designed for Japanese youth and was developed by Atsushi Oshio et al. [14].

\subsection{Analytical Method}

Forced entry multiple regression analysis was carried out using four subdomains of norm-consciousness and three subdomains of resilience as the independent variables and nine risk behaviors as the dependent variables. In this case, the responses that indicate more preferable conditions of norm-consciousness and resilience were given higher scores: $5=$ Think very so and $1=$ Do not think very so to the item "It is necessary to observe the national laws". No point was given when risk behavior was observed, however otherwise 1 point was given. The significance level in statistics was assumed to be 5\%. A statistical package IBM SPSS Statistics 19 was used.

\section{RESULTS}

In the multiple regression analysis using nine risk behaviors as the dependent variables, adjusted $\mathrm{R}^{2}$ was all significant for both males and females (Table 1).

For males, the subdomains of norm-consciousness showed significant positive standardized partial regression 


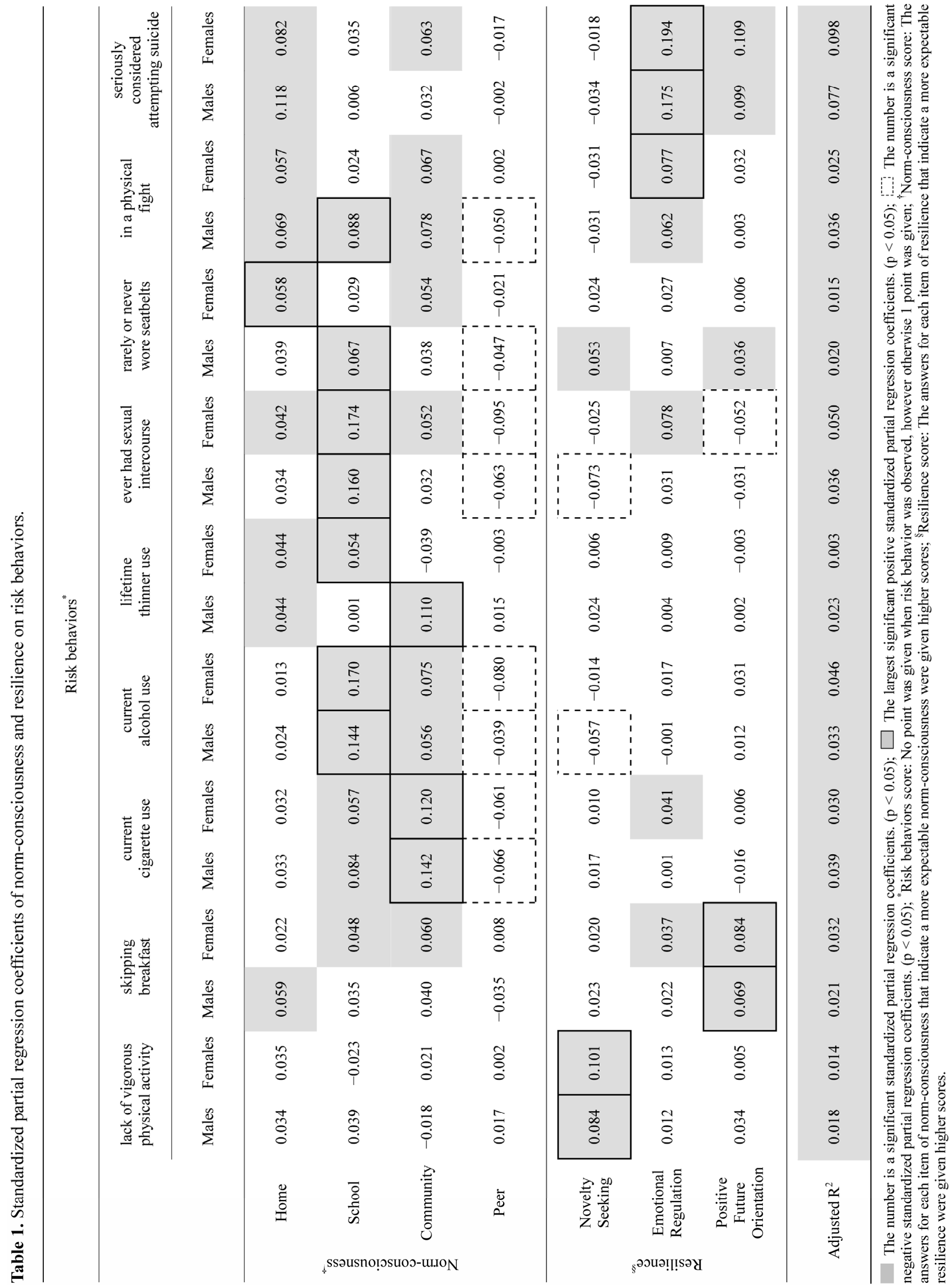


coefficients for all risk behaviors except for "lack of vigorous physical activity". In particular, "current alcohol use", "ever had sexual intercourse", "rarely or never wore seatbelts", and "in a physical fight" showed the largest standardized partial regression coefficients for the norm-consciousness in school. "Current cigarette use" and "lifetime thinner use" showed the largest standardized partial regression coefficients for the norm-consciousness in community. The subdomains of resilience showed significant positive standardized partial regression coefficients for five risk behaviors. "Lack of vigorous physical activity" showed the largest standardized partial regression coefficient for Novelty Seeking, "skipping breakfast" showed that for Positive Future Orientation, and "seriously considered attempting suicide" showed that for Emotional Regulation.

The results for females were almost similar to those for males. That is, the subdomains of norm-consciousness showed significant positive standardized partial regression coefficients for all risk behaviors except for "lack of vigorous physical activity". In particular, "current alcohol use", "lifetime thinner use", and "ever had sexual intercourse" showed the largest standardized partial regression coefficients for the norm-consciousness in school, "current cigarette use" showed that for the normconsciousness in community, "rarely or never wore seatbelts" showed that for the norm-consciousness at home. The subdomains of resilience showed significant positive standardized partial regression coefficients for six risk behaviors. "Lack of vigorous physical activity" showed the largest standardized partial regression coefficient for Novelty Seeking, "skipping breakfast" showed that for Positive Future Orientation, and "in a physical fight" and "seriously considered attempting suicide" showed that for Emotional Regulation.

In contrast, the norm-consciousness among peers showed significant negative standardized partial regression coefficients for "current cigarette use", "current alcohol use", and "ever had sexual intercourse" for both males and females, and for "rarely or never wore seatbelts" and "in a physical fight" for males only. Regarding the subdomains of resilience, "current alcohol use" and "ever had sexual intercourse" showed significant negative standardized partial regression coefficients for Novelty Seeking for males and "ever had sexual intercourse" showed that for Positive Future Orientation for females.

\section{DISCUSSION}

It has been pointed out that various factors are related to risk behaviors. In this study, we focused on normconsciousness and resilience of adolescents in Japan and examined their influences on the prevalence of youth risk behaviors.

Recently, there have been studies that considered the relationship between risk behaviors and norm-consciousness among adolescents in Japan. For instance, Chie Kataoka et al. [7] suggested that the increased norm-consciousness contributed to the comprehensive prevention of risk behaviors including behaviors that violate laws and regulations (e.g., cigarette smoking, alcohol drinking, drug abuse, and traffic-related behaviors). Moreover, they examined the influence of norm-consciousness relative to that of self-esteem, which has been reported as a factor related to risk behaviors by many researchers including those from Western countries, US, and Japan, and reported that norm-consciousness, particularly the norm-consciousness in school, was strongly related to risk behaviors of high school students.

In this study, we compared the influences of normconsciousness with those of resilience, and found that norm-consciousness was strongly related to risk behaviors. Concretely, all risk behaviors except for "lack of vigorous physical activity" showed relationships with the norm-consciousness in school, community, or at home for both males and females. Among these results, "current cigarette use", "current alcohol use", "lifetime thinner use", "ever had sexual intercourse", and "rarely or never wore seatbelts" showed stronger relationships with the subdomains of norm-consciousness including that in school than with the subdomains of resilience for both males and females. From these results, it was suggested that the increase in norm-consciousness including that in school is very important to comprehensively prevent youth risk behaviors.

There are clear laws and rules for group living at home and in schools and local communities where adolescents are closely related to in their daily lives. Unwritten rules and promises may also arise among people. Norm-consciousness, a respect for norms to maintain the public order and good human relationships and for the senses of values among adults at home and in schools and local communities, is important for adolescents in their developmental stage to make appropriate decisions on their behaviors. Norm-consciousness of adolescents is enhanced through human relationship, various experiences, and learning in schools. It will be required to clarify how to promote the enhancement of adolescents' norm-consciousness, particularly the norm-consciousness in school, as indicated to be strongly related to risk behaviors, in education to prevent risk behaviors in the future. Note that adolescents in their developmental stage tend to temporarily have a rebellious attitude. The enhancement of adolescents' norm-consciousness is not promoted when students are forced to observe rules and regulations and accept one-sided beliefs of the adults at home and in schools and local communities. 
However, the results indicated that the increase in the norm-consciousness among peers does not necessarily lead to the prevention of risk behaviors. Rather, the increased norm-consciousness among peers encouraged cigarette smoking, alcohol drinking, and sexual behaviors for both males and females, which supports the previous finding [7]. At this point, the norm-consciousness among peers is not only negative toward risk behaviors but also might be affirmative toward them.

Relationships between youth risk behaviors and resilience have been recently reported with the results of large-scale surveys in the US. According to the Youth Risk and Resilience Survey [15] in New Mexico, the prevalences of risk behaviors such as cigarette smoking, alcohol drinking, drug abuse, violence, and self-harm behaviors were low among students with high resilience. Moreover, the report [16] of California Healthy Kids Survey showed that resilience is related to the simultaneous prevalence of multiple risk behaviors such as cigarette smoking, alcohol drinking, eating vegetables and fruits, and lack of physical activity. These findings suggest that resilience is a factor to be emphasized in the prevention of youth risk behaviors.

Also in this study for Japanese adolescents, it was suggested that the increased resilience contributes to the prevention of specific risk behaviors for both males and females. Particularly, "seriously considered attempting suicide" showed the strongest correlation with Emotional Regulation for both males and females. Attention is required because suicide is the leading cause of death among Japanese adolescents aged 15 - 19 years and accounts for more than $30 \%$ [17]. "Lack of vigorous physical activity" and "skipping breakfast" showed strong correlations with the subdomains of resilience. Therefore, it is important to enhance the resilience of adolescents also in the formation and maintenance of their fundamental healthy lifestyle.

In this study, any subdomain of resilience was not related to prevent of "lifetime thinner use" and "current alcohol use" for both males and females, "current cigarette use" and "ever had sexual intercourse" for males only, and "rarely or never wore seatbelts" for females only. More amounts of data should be further collected and examined in the future.

In conclusion, norm-consciousness is an important protective factor to comprehensively prevent risk behaviors among Japanese adolescents compared with resilience. It is expected to be effective to enhance the normconsciousness particularly in school and community. In addition, resilience should also be emphasized as a factor to prevent specific risk behaviors.

\section{ACKNOWLEDGEMENTS}

The authors would like to thank the school staff members and stu- dents who cooperated in this study. This work was supported by JSPS KAKENHI (22500622).

\section{REFERENCES}

[1] Centers for Disease Control and Prevention (2010) Youth risk behavior surveillance-United States, 2009. Morbidity and Mortality Weekly Report, 59, 1-142.

[2] Mirestean, N. and Irimie, S.I. (2010) Aspects of tobacco, alcohol and drugs consumption among students from Romania. 20th International Union for Health Promotion and Education World Conference on Health Promotion, Geneva, 11-15 July 2010.

[3] Health Promotion Research and Development Unit of the Medical Research Council, South Africa (2010) The 2nd South African National Youth Risk Behaviour Survey 2008.

[4] Park, S.-W. (2010) Surveillance systems for health risk behaviors and health levels among adolescents in Korea. Japanese Journal of School Health, 52, 116-124 (in Japanese).

[5] Nozu, Y., Watanabe, M., et al. (2006) Youth risk behavior among Japanese high school students: Results of the 2001 national survey. Japanese Journal of School Health, 48, 430-447 (in Japanese).

[6] Ichimura, K., Shimomura, Y. and Watanabe, M. (2001) Harmful substance abuse, cigarette smoking, alcohol drinking and perceived norms among junior and senior high school students. Japanese Journal of School Health, 43, 39-49 (in Japanese).

[7] Kataoka, C., Nozu, Y., Kubo, M., Sato, Y. and Watanabe, M. (2010) Relative influence of self-esteem and normconsciousness on prevalence of youth risk behavior among Japanese high school students. School Health, 6, 6-11.

[8] Mita, M. (1966) Theory of value consciousness. Kobundo Publishers Inc., Tokyo (in Japanese).

[9] Masten, A.S., Best, K.M. and Garmezy, N. (1990) Resilience and development: Contributions from the study of children who overcome adversity. Development and Psychopathology, 2, 425-444. doi:10.1017/S0954579400005812

[10] Mark, W.F. (2004) Risk and resilience in childhood: An ecological perspective. 2nd Edition, NASW Press, Washington DC.

[11] Ministry of Health, Labour and Welfare (2011) Annual health, labour and welfare report 2009-2010 (in Japanese). http://www.mhlw.go.jp/english/wp/wp-hw4/index.html

[12] Statistics Bureau (2010) Annual report on the labour force survey (in Japanese).

http://www.stat.go.jp/english/data/roudou/report/2010/ind ex.htm

[13] Uehara, C., Nozu, Y., Kubo, M., Sato, Y. and Watanabe, M. (2008) Reliability and validity of norm-consciousness scale for youth risk behavior. Japanese Journal of School Health, 50, 159-165 (in Japanese).

[14] Oshio, A., Nakata, M., Kaneko, H. and Nagamine, S. (2002) Development and validation of an adolescent re- 
silience scale. Japanese Journal of Counseling Science, 35, 57-65 (in Japanese).

[15] Dan, G. (2010) Highlights from the 2009 New Mexico high school youth risk and resiliency survey.

http://nmhealth.org/ERD/HealthData/pdf/ER\%20YRRS\% 20092410.pdf

[16] Ritesh, M., William, J.M., Antronette, K.Y., Yao, L. and
Minal, P. (2009) Resilience and patterns of health risk behaviors in California adolescents. Preventive Medicine, 48, 291-297. doi:10.1016/j.ypmed.2008.12.013

[17] Cabinet Office, Government of Japan (2011) Annual reports on suicide prevention (in Japanese).

http://www8.cao.go.jp/jisatsutaisaku/whitepaper/w-2011/ $\underline{\mathrm{html} / \text { index.html }}$ 